\title{
Article: Beta diversity patterns derived from island biogeography theory
}

$$
\text { Muyang } \mathrm{Lu}^{*}{ }^{1} \text {, David Vasseur }{ }^{1} \text {, Walter Jetz }{ }^{1,2}
$$

3

4 1. Department of Ecology and Evolutionary Biology, Yale University, 165 Prospect

5 Street, New Haven, CT, 06520, USA.

6 2. Department of Life Sciences, Imperial College London, Silwood Park Campus,

$7 \quad$ Buckhurst Road, SL5 7PY Ascot, Berks, UK.

8

$9 \quad$ Running head: beta diversity of islands

10 Words in the abstract: 164

11 Words in main body: 3820

12 References: 85

13 Table: 1

14 Figure: 3

$15 *$ Correspondence: Muyang Lu (email: muyang.lu@yale.edu) 


\section{Abstract}

17 The Theory of Island Biogeography (TIB) has been successful in predicting alpha

18 diversity patterns such as species-area relationships and species-abundance

19 distributions. Although beta diversity (i.e. the dissimilarity of community composition)

20 has long been recognized as an important element of the TIB and is crucial for

21 understanding community assembly processes, it has never been formally

22 incorporated into the theory. Here we derive theoretical predictions for the expected

23 pairwise beta diversity values under a species-level neutral scenario where all species

24 have equal colonization and extinction rates. We test these predictions for the avian

25 community composition of 42 islands (and 93 species) in the Thousand Island Lake,

26 China. We find that alpha diversity patterns alone do not distinguish a species-level

27 neutral model from a non-neutral model. In contrast, beta diversity patterns clearly

28 reject a species-level neutral model. We suggest that the presented theoretical

29 integration beta diversity offers a powerful path for testing the presence of neutral

30 processes in ecology and biogeography.

31

32 Keywords: neutral theory; null model; beta diversity; meta-community; incidence

33 function; community assembly; regional species pool 


\section{INTRODUCTION}

35 Island biogeography is undergoing a renaissance (Warren et al. 2015; Santos et al. 2016; Patiño et al. 2017), at the heart of which lies the equilibrium theory of island biogeography (TIB) formulated by MacArthur and Wilson 50 years ago (1967). The TIB is most celebrated for elegantly linking species richness with colonization and extinction rates, which in turn are influenced by island area and distance to the mainland. Equilibrium TIB makes two key predictions: 1) equilibrium species

41 richness depends on colonization and extinction rates; 2) community composition of an island is undergoing constant turnover when at equilibrium. While early empirical studies have focused on testing the equilibrium and temporal turnover predictions May 1977), recent interest in TIB is stimulated by the success of Hubbell's unified neutral theory (a descendant of the TIB; Hubbell 2001), the advance of food web theories (Holt 2009), the developments of phylogenetic analyses (Valente et al. 2015; Lim and Marshall 2017), and the accumulation of island datasets (Weigelt et al. 2013, 2016). Motivated by the desire for a general island biogeography theory, more and more complexities have been added to the original framework, including allometric

51 scaling (Jacquet et al. 2017), trophic interactions (Gravel et al. 2011; Cazelles et al.

52 2016), speciation (Chen and He 2009; Rosindell and Phillimore 2011; Rosindell and

53 Harmon 2013), habitat heterogeneity (Kadmon and Allouche 2007), and island

54 ontogenies (Whittaker et al. 2008; Borregaard et al. 2016). While the complexity of 
55 the theory has increased, examinations to date have focused on alpha diversity

56 patterns such as species richness (MacArthur and Wilson 1967), species-abundance

57 relationships (Rosindell and Phillimore 2011; Rosindell and Harmon 2013; Kessler

58 and Shnerb 2015), functional diversity (Jacquet et al. 2017), and endemism (Chen and

59 He 2009).

60 One particular omission in the theory and tests of the TIB to date is beta diversity,

61 i.e. the compositional dissimilarities among communities, a widely used metric in

62 biodiversity studies (Leprieur et al. 2011; Stegen et al. 2013; Segre et al. 2014; Si et al.

63 2016). The integration of beta diversity patterns into the TIB has the potential to

64 unlock a range of uses in community assembly studies and the development of a

65 unified meta-community theory (Leibold et al. 2004).

66

67 The concept of beta diversity was first introduced by Whittaker $(1960,1965)$ and

68 was quickly linked to an array of important mechanisms in community assembly:

69 biotic interaction (Graham and Fine 2008), environment filtering (Veech and Crist

70 2007; Buckley and Jetz 2008), dispersal limitation (Ojima and Jiang 2017; Wu et al.

71 2017), and historical contingency (Fukami and Nakajima 2011). However, despite the

72 widespread recognition of the beta diversity concept, there is little consensus about

73 the best way to measure it (Tuomisto 2010a, 2010b; Anderson et al. 2011).

74 Depending on the purpose of a study, beta diversity could be measured as turnover

75 (directional) or variation (non-directional) of compositional similarity (Nekola and 
76 White 1999; Legendre and De Cáceres 2013); based on pairwise or multiple-sites

77 comparisons (Hui and McGeoch 2014; Arita 2017; Latombe et al. 2017; Marion et al.

78 2017); decomposed in additive or multiplicative framework (with respect to alpha and

79 gamma diversity; Jost 2007; Cabral et al. 2014); calculated by incidence

80 (presence-absence) or abundance data (Chao et al. 2016). Even for the commonly

81 used pairwise beta diversity, there is a heated debate about what is the best way to

82 partition it into richness difference and replacement (or nestedness and turnover)

83 components (Baselga 2010; Podani and Schmera 2011; Legendre 2014; Baselga and

84 Leprieur 2015). It is generally agreed that different measures quantify different

85 aspects of communities and that their use should be guided by the question asked

86 (Anderson et al. 2011; Legendre 2014). Although beta-diversity measures have been

87 increasingly synthesized under more general frameworks such as Hill's number and

88 variance of community matrix (Chao et al. 2016), only a few of them have been

89 examined analytically from process-based theories (Chave and Leigh 2002; Condit et

90 al. 2002; Zillio et al. 2005; Connolly et al. 2017). Moreover, theoretical

91 considerations of beta-diversity patterns to date have been abundance-based and

92 hence limited to more restricted datasets, such as vegetation plots (Chave and Leigh

93 2002; Condit et al. 2002; Zillio et al. 2005). In summary, the link between beta

94 diversity patterns and fundamental processes such as colonization and extinction in

95 the TIB remain ill developed. 
97 In this study, we set out to address this shortcoming and provide a theoretical and

98 example empirical integration of beta diversity into TIB. Specifically, we present the

99 first theoretical predictions for the expected pairwise beta diversity patterns for both

100 Jaccard and Sørensen indices in the classic MacArthur and Wilson framework. This

101 framework constitutes in essence a species-level neutral model (as opposed to the

102 individual-level neutral theory; Hubbell 2009; Rosindell and Phillimore 2011). The

103 species-level neutral model assumes that all species have the same colonization and

104 extinction rates, while the individual-level neutral model assumes that all individuals

105 have the same colonization and extinction rates, which allows species-level

106 asymmetry to arise because of different abundance (Rosindell and Harmon 2013). We

107 first derived the probability mass function and expected value of pairwise beta

108 diversity in a general case where the equilibrium assumption is not required. We then

109 examined our results under the classic MacArthur-Wilson equilibrium framework.

110 Finally, we test the derived predictions for a dataset of 93 avian species occurring

111 across 42 islands of the Thousand Island Lake Region in China (Appendix, Wang et

112 al. 2010).

113

114 MATERIAL AND METHODS

115 To derive expected beta diversity, we need to consider the joint statistical distribution

116 of multiple islands. We here focus on pairwise community comparisons, because

117 average pairwise dissimilarity is shown to be the only unbiased and consistent 
118 estimator when applied to empirical data (Marion et al. 2017). We also calculated the

119 expected value of the partitioned components of pairwise beta dissimilarity for both

120 Baselga and Podani families (Baselga 2010; Podani and Schmera 2011). To test our

121 predictions, we applied an "Incidence Function" approach pioneered by Diamond

122 (1975) and used by Connor and Simberloff $(1978,1979)$ during early debates of null

123 models.

\section{Probability mass function of pairwise beta diversity}

126 All pairwise beta diversity indices share the same probability mass function to

127 describe island community composition because they are calculated from four

128 quantities: the total number of species of two islands $N$ (regional species richness), the

129 number of species shared by both islands $k$, the number of species unique to the first

130 island $i$, and the number of species unique to the second island $j$ (thus $N=k+i+j$ ).

131 Then the number of species present on the first island is $i+k$, and the number of

132 species present on the second island is $j+k$.

133 Denoting the size of the mainland species pool as $M$, the probability mass function of

134 pairwise beta diversity, conditioning on $N, k$ and $i$, can be derived as follows: let the

135 occurrence probability of the $i^{\text {th }}$ species on the first island be $p$, and the $j^{\text {th }}$ species on

136 the second island be $q$. Furthermore, assuming that the occurrence probabilities are

137 properties of the island rather than of species, the probability of obtaining exactly $i$

138 unique species and $k$ shared species from a regional pool of size $M$ is given by 
$139 p^{k+i}(1-p)^{M-(k+i)}$. Similarly, the probability of obtaining $j$ unique and $k$ shared species on

140 the second island is $q^{k+j}(1-q)^{M-(k+j)}$, which yields the probability of obtaining the

141 regional community specified by $i, j, k$ and $M$, as $p^{k+i}(1-p)^{M-(k+i)} q^{k+j}(1-q)^{M-(k+j)}$.

142 Assuming that species are neutral with respect to their occurrence probabilities

143 within an island, we then count how many different combinations of island

144 communities could be obtained with regional richness $N, k$ shared species, and $i$

145 unique species to the first island (the number of unique species to the second island is

146 then fixed as $k-i)$. There are exactly $\left(\begin{array}{l}M \\ N\end{array}\right)$ ways of choosing $N$ out of $M$ species to be

147 present in either or both islands from the mainland species pool. Similarly, there are

$148\left(\begin{array}{l}N \\ k\end{array}\right)$ ways to choose $k$ out of $N$ species to be shared by both islands and for the unique

$149 N-k$ species, there are $\left(\begin{array}{c}N-k \\ i\end{array}\right)$ ways of assigning $i$ species to the first island. After

150 simplification, the probability of pairwise beta diversity conditioning on the total

151 number of species of both islands $N$, the number of species shared by both islands $k$,

152 and the number of species unique to the first island $i$ is:

$153 \operatorname{Pr}$ (regional.community $\mid N, k, i)=\left(\begin{array}{c}M \\ N\end{array}\right)\left(\begin{array}{c}N \\ k, i\end{array}\right) p^{k+i} q^{k+j}(1-p)^{M-(k+i)}(1-q)^{M-(k+j)}$

156 Because beta diversity measures (e.g. Jaccard and Sørensen indices) are usually

157 undefined when $N=0$, the expected pairwise beta diversity should be normalized by

158 the term 1- $(1-p)^{M}(1-q)^{M}$ to exclude "double-absence" scenarios (Anderson et al.

159 2011). Only the expected Jaccard dissimilarity (Jaccard dissimilarity is defined as 
$1601-\frac{k}{N}$ ) has a simple analytical form that is independent of the size of mainland

161 species pool $M$ (see Appendix S1 in Supporting Information for derivation; we do not

162 show the analytical form of the expected Sørensen dissimilarity because the specific

163 form depends on the size of mainland species pool):

$164 E($ Jaccard.dissimilarity $)=\frac{p+q-2 p q}{p+q-p q}$

166 It has a form that is similar to the expected pairwise Jaccard derived by Chase et al.

167 (2011) but allows two islands to have different occurrence probabilities. $p$ and $q$ could

168 also be interpreted as probabilities of the same island at different times, and in that

169 case (2) becomes the expected temporal Jaccard dissimilarity. This quantity does not

170 require any equilibrium assumptions of alpha diversity and hence could be seen as a

171 generalized version of the apparent turnover derived by Diamond and May (1977)

172 which is essentially a pairwise Sørensen dissimilarity index in equilibrium (Morrison

173 2017).

174 We also calculated the expected pairwise beta diversity for Jaccard and Sørensen

175 families as well as their partitioned components (for detailed formulas of the Baselga

176 and Podani families see Baselga \& Leprieur 2015) conditioning on both islands

177 having species $(i+k>0$ and $j+k>0)$ because empty islands are sometimes excluded

178 from analysis (Wang et al. 2016). In Baselga's family, pairwise dissimilarity is

179 partitioned into the nestedness component and the turnover component. The

180 nestedness component measures the extent to which species in a smaller community 
181 are a subset of species in a larger community, while the turnover component measures

182 how much of the dissimilarity is caused by species replacement (Baselga 2010). The

183 turnover component and the nestedness component in Baselga's family, respectively,

184 correspond to the replacement component and the richness difference component in

185 Podani’s family (Legendre 2014).

187 Island biogeography theory

188 Following the stochastic version of the TIB (MacArthur and Wilson 1967), the

189 occurrence probability of a species on an island is a function of colonization rate $c$

190 and extinction rate $e$ :

$191 \quad \frac{d p}{d t}=c(1-p)-e p$

192 When occurrence probability of a species is at stochastic equilibrium (stationary

193 distribution), the occurrence probability is:

194

$p_{i, e q}=\frac{c}{c+e}$

$195 \operatorname{Let} \theta=\frac{e}{c}$, which is the relative extinction rates, equation (4) becomes:

$196 p_{i, e q}=\frac{1}{1+\theta}$

197 Substitute $p=q=\frac{1}{1+\theta}$ into equation (2), the expected Jaccard dissimilarity when

198 two islands have equal colonization and extinction probabilities is:

$199 E($ Jaccard. dissimilarity $)=\frac{2 \theta}{1+2 \theta}$

200 To take into account asymmetry between islands (e.g. the effect of area, isolation or

201 habitat types), let $p=\frac{1}{1+\theta}$ and $q=\frac{1}{1+z \theta}$, so that the relative extinction rate on the 
202 second island is $z$ times the relative extinction rate on the first island. Equation (2)

203 becomes:

$204 E($ Jaccard.dissimilarity $)=\frac{(1+z) \theta}{1+(1+z) \theta}$

206 Empirical tests

207 Data

208 We use a published dataset of avian community composition for 93 birds and 42

209 islands in The Thousand Island Lake, China to test our predictions (see Appendix in

210 Wang et al. 2010). The region $\left(29^{\circ} 22^{\prime \prime}-29^{\circ} 50^{\prime \prime} \mathrm{N}, 118^{\circ} 34^{\prime \prime}-119^{\circ} 15^{\prime \prime} \mathrm{E}\right)$ consists of

211 an inundated lake with more than 1000 islands created by dam constructions in 1959 .

212 Because the islands were formed recently, there was no in situ speciation in this

213 region. The relative small area of the region $\left(573 \mathrm{~km}^{2}\right)$ ensures that the islands are

214 accessible to most of the bird species (Si et al. 2016). Bird occupancies from 2006 to

2152009 on 42 islands were documented using line-transect method. Island variables

216 measured in the dataset include area, distance to mainland, habitat diversity and plant

217 species richness.

\section{Incidence function and statistical analysis}

220 Because extinction rates and colonization rates are difficult to measure directly, an

221 alternative way is to use an "Incidence function" approach to estimate parameters

222 from a snapshot of occupancy patterns (Diamond 1975; Hanski 2009). To test the 
223 predictions of island biogeography theory, equation (4) is modeled as a function of

224 isolation and area. Parameters are fitted by maximum likelihood with binomial

225 distribution. Three neutral models and one non-neutral model are examined in this

226 study:

227 Neutral model 1: Inverse ratio

228 Colonization rates are modeled as an inverse ratio function of isolation: $c=\frac{a}{I}$, and 229 extinction rates are modeled as an inverse ratio of area: $e=\frac{b}{A} \cdot a$ and $b$ are fitted 230 parameters.

231 Neutral model 2: Exponential

232 Colonization rates are modeled as an exponential function of isolation:

$233 c=\exp (-a I)$, and extinction rates are modeled as an exponential function of area:

$234 e=\exp (-b A) . a$ and $b$ are fitted parameters. Substitute $c=\exp (-a I)$ and

$235 e=\exp (-b A)$ into equation (4), the occurrence probability becomes

$236 p=\frac{1}{1+\exp (-b A+a I)}$. Thus the exponential neutral model is equivalent to a logistic

237 regression without intercepts, which could be directly fitted by 'glm' in R with

238 binomial distribution and logit link.

239 Neutral model 3: GLM

240 This model adds habitat diversity and plant richness to the predictors in addition to

241 area and isolation in Neutral Model 2. AIC is used to select the best model. Area and

242 isolation are log-transformed to ensure better linearity.

243 Non-neutral model: Aggregate species-level GLM 
244 To take into account species-level non-neutrality, species identity is included as a

245 fixed effect categorical variable in the GLM, which allows each species to have a

246 different intercept (baseline occurrence probability) but share the same response to

247 island area, isolation, plant richness and habitat types. This is essentially a stacked

248 species distribution modeling approach (Calabrese et al. 2014; Ko et al. 2016), which

249 increases the number of parameters by the number of species minus one ( 92

250 parameters in this case). This procedure aims to capture the observed

251 presence-absence variation among species but does not pin down the causes of

252 species-level non-neutrality such as traits and abundance differences. More realistic

253 assumptions of non-neutrality such as different responses to area and isolation could

254 be made but are not the main focus of this paper. AUC and AIC values are calculated

255 to compare the overall performance of the models. AUC is calculated with a

256 Mann-Whitney U statistic using R package 'PresenceAbsence'. AIC is calculated

257 from the best-fitted likelihood function. $\triangle \mathrm{AIC}$ are calculated by subtracting the AIC

258 of the null model (GLM with only one intercept) from the AIC of the fitted model.

260 Predictions of alpha diversity and beta diversity

261 The predicted species richness of each island is given by the summed fitted

262 occurrence probabilities of all species as used in conventional stacked species

263 distribution modeling (Calabrese et al. 2014; Ko et al. 2016). While predicted

264 pairwise Jaccard dissimilarity can be calculated analytically from equation (2), the 
265 partitioned nestedness and turnover components can only be estimated by simulations

266 (we only present the results of Baselga's family because its partitioned components

267 are independent with each other when gamma diversity is fixed; Baselga \& Leprieur

268 2015). We therefore estimate predicted pairwise beta diversity as the mean of 1000

269 community matrices simulated from the fitted occurrence probabilities. Observed

270 values are regressed against predicted values using ordinary least square. $R^{2}$ is used as

271 a measure of predictive power for alpha and beta diversity patterns. If the model

272 predicts the observed patterns well, the fitted regression line should be close to the $1: 1$

273 ratio line when observed values are plotted against predicted values. All statistical

274 analyses are performed in R 3.3.2.

275

276 RESULTS

277 Symmetric islands (same colonization and extinction rates for both islands)

278 The expected Jaccard dissimilarity conditional on both islands having non-zero

279 species richness increases monotonically with relative extinction rate $\theta$ and the size

280 (number of species) of the mainland species pool $M$. As $M$ increases, the expected

281 Jaccard dissimilarity converges to $\frac{2 \theta}{1+2 \theta}$ (Fig. 1a). When decomposed into turnover 282 and nestedness (or replacement and richness difference) components, contrasting 283 patterns are observed: while the turnover component and the replacement component 284 increase monotonically with $\theta$ and $M$ (Fig. 1b, e), the nestedness component and 285 richness difference component are both unimodal functions of $\theta$ with maximum 
286 values less than 0.3 (Fig. 1c, f). The maximum nestedness decreases as $M$ increases

287 (Fig. 1c), while the maximum richness difference changes little with the increase of $M$

288 (Fig. 1f). The relative importance of turnover increases monotonically with $\theta$ and $M$

289 (Fig. 1d). In a special case of 2 mainland species, the ratio of expected turnover and 290 expected nestedness equals $\theta$ (Fig. 1d). The Sørensen family indices have similar 291 quantitative behaviors as the Jaccard family indices (see Fig. S1 in Appendix).

\section{Asymmetric islands (different colonization and extinction rates for two islands)}

294 The expected Jaccard dissimilarity conditional on both islands having non-zero 295 species richness converges to $\frac{(1+z) \theta}{1+(1+z) \theta}$ as $M$ increases. But the deviation from $296 \frac{(1+z) \theta}{1+(1+z) \theta}$ also increases with islands asymmetry $z$ (Fig. 2a, e, i). The turnover 297 component increases monotonically with $\theta$ and $M$, and decreases with $z$ (Fig. 2b, f, j).

298 The nestedness component decreases with $M$ when $z$ is small (Fig. 2c), but this 299 relationship with $M$ is reversed when $z$ gets larger (Fig. $2 \mathrm{~g}, \mathrm{k}$ ). The ratio of expected 300 turnover and expected nestedness also increases with $M$ when $z$ is small (Fig. 2d), and 301 decreases with $M$ when $z$ is large (Fig. 2h, 1).

\section{Empirical test}

304 We tested the above predictions for an inland lake island system using 93 bird species 305 and 42 islands. We used an incidence function approach to fit the observed occupancy 
patterns and calculated predicted alpha and beta diversity patterns from the fitted models. The non-neutral GLM has the lowest AIC and highest AUC in all models

311 systematically underestimate the nestedness component, but overestimate the turnover

314 predictions, yet it overestimates pairwise beta diversity at the higher range of the predictions (Fig. 3a-d). Both the neutral GLM and the non-neutral GLM successfully predict the observed alpha diversity pattern (Fig. 3i, m), but only the non-neutral GLM successfully predicts the observed beta diversity patterns (Fig. 3n-p).

\section{DISCUSSION}

\section{Theoretical results}

322 We derived a set of novel predictions of beta diversity patterns from the island

323 biogeography theory. Under the classic MacArthur and Wilson framework, pairwise

324 beta diversity patterns are determined by three factors: the size of the mainland species pool, extinction rates and colonization rates. Conditional on at least one island having species $(N>0)$, only the expected Jaccard dissimilarity is independent of the 
328 richness $(i+k>0$ and $j+k>0)$, all indices are dependent on the size of mainland

329 species pool. Ignoring empty islands has a huge influence on beta diversity patterns

330 when the size of mainland species pool is small (e.g. less than 10 species), but the

331 effect becomes negligible toward larger mainland species pool sizes, because of the

332 rapidly decreasing probability of empty islands (Fig. 1a). This result stresses the need

333 to include empty islands in empirical tests of the island biogeography theory (Wang et

334 al. 2016).

336 Pairwise Jaccard dissimilarity increases with relative extinction rates (the ratio 337 between extinction rates and colonization rates). This is because when extinction 338 events become more frequent the chance of forming "checkerboard" patterns 339 (Diamond 1975) grows, as shown by the increasing turnover component (Fig. 1b).

340 The nestedness component first increases then decreases with relative extinction rates

341 (Fig. 1c). The maximum nestedness is achieved when extinction rates are less than 342 colonization rates (Fig. 1c). When relative extinction rates or the size of mainland 343 species pool gets larger, the increase of Jaccard dissimilarity is mainly driven by the 344 increasing turnover component (Fig. 1d). Indices in Podani's family (the replacement 345 and richness difference components) have similar qualitative behaviors, consistent 346 with previous finding that the partitioned components in Baslega's family are 347 correlated with the partitioned components in Podani's family (Legendre 2014; 348 Baselga and Leprieur 2015). Island asymmetry, which takes into account the effect of 
349 isolation and area, does not change the qualitative behaviors of the indices (Fig 2), but

350 increases the level of nestedness, because species in a small (distant) island are more

351 likely to be a subset of species in a large (near) island.

353 Neutral theory and null models

354 Our theoretical results are derived from a species-level neutral theory. We show that

355 both the neutral GLM and the non-neutral GLM are successful in predicting species

356 richness (alpha diversity) of birds in the Thousand Islands Lake (Fig. 3i, m). But only

357 the non-neutral model predicts the observed beta diversity patterns. The patterns of

358 partitioned components further reveal that the neutral GLM fails to predict the

359 observed pairwise Jaccard dissimilarity because it overestimates turnover and

360 underestimates nestedness (Fig. 3j-1). Our analysis does not include biological factors

361 that may cause species-level non-neutrality, but the presented framework is flexible to

362 include such information if available.

363 Null models have become one of the most important tools in ecology. Despite their

364 increasing popularity, it has also been recognized that their assumptions (e.g. the

365 widely used random shuffling approaches) can have serious limitations, especially in

366 the presence of multiple processes (Gotelli and Ulrich 2012; Pigot and Etienne 2015;

367 Miller et al. 2017; O’Dwyer et al. 2017). In terms of beta diversity, the entangled

368 links among alpha, beta and gamma diversity are known to reduce the statistical

369 power of randomization tests and generate ambiguity in their interpretations (Chase et 
370 al. 2011; Kraft et al. 2011; Qian et al. 2012, 2013; Ulrich et al. 2017). Our results

371 support the use of mechanistic null models such as those based on neutral theory

372 (O’Dwyer et al. 2009; Rosindell et al. 2012), maximum entropy theory (Xiao et al.

373 2015, 2016; O’Dwyer et al. 2017), and incidence functions (Hanski et al. 1996; Helm

374 et al. 2006; Hanski 2009) to improve upon random-shuffling null models in

375 hypothesis testing. These mechanistic models could generate multiple diagnostic

376 patterns and allows stronger test for ecological theories.

Future directions

379 Our results are derived from a mainland-island model where the colonization and

extinction events on islands are independent of one another. More realistic

381 modifications should be considered in order to predict biodiversity changes in a real

382 landscape (Hanski 2009). For example, environmental heterogeneity and local

383 dispersal are found to be important drivers of beta diversity in experimental studies

384 (Grainger and Gilbert 2016; Gianuca et al. 2017; Ojima and Jiang 2017; Rodrigues

385 and Diniz-Filho 2017). Moreover, in oceanic islands, high endemism arises from

386 speciation events (Chen and He 2009; Gascuel et al. 2016; Steinbauer et al. 2016),

387 which are likely to increase the level of species turnover. Future studies should also

388 extend presence-based measures to abundance-based beta diversity measures which

389 may provide additional insights into the dynamics of meta-community (Bashan et al.

390 2016; Kalyuzhny and Shnerb 2017). We believe that the integration of beta diversity 
391 patterns into the Theory of Island Biogeography offers new opportunities to infer

392 community assembly processes.

\section{ACKNOWLEDGEMENTS}

395 We thank Dr. James Rosindell for providing helpful comments for earlier drafts. We

396 thank members in Jetz Lab and Vasseur Lab for feedbacks and dicussions.

\section{AUTHORSHIP}

398 ML and DV conceived of the idea. The idea was further developed with DV and WJ's 399 input. ML conducted the analyses and wrote the manuscript. All authors assisted with 400 revisions.

\section{REFERENCE}

402 Anderson, M. J., T. O. Crist, J. M. Chase, M. Vellend, B. D. Inouye, A. L. Freestone, roadmap for the practicing ecologist. Ecology Letters 14:19-28.

Arita, H. T. 2017. Multisite and multispecies measures of overlap, co-occurrence, and co-diversity. Ecography 40:709-718.

407 Baselga, A. 2010. Partitioning the turnover and nestedness components of beta diversity. Global Ecology and Biogeography 19:134-143.

409 Baselga, A., and F. Leprieur. 2015. Comparing methods to separate components of beta diversity. Methods in Ecology and Evolution 6:1069-1079.

411 Bashan, A., T. E. Gibson, J. Friedman, V. J. Carey, S. T. Weiss, E. L. Hohmann, and 

262.

414 Borregaard, M. K., T. J. Matthews, and R. J. Whittaker. 2016. The general dynamic model: towards a unified theory of island biogeography? Global Ecology and Biogeography 25:805-816.

417 Buckley, L. B., and W. Jetz. 2008. Linking global turnover of species and environments. Proceedings of the National Academy of Sciences 105:1783617841.

Cabral, J. S., P. Weigelt, W. D. Kissling, and H. Kreft. 2014. Biogeographic, climatic and spatial drivers differentially affect $\alpha$-, $\beta$ - and $\gamma$-diversities on oceanic $281: 20133246$

Calabrese, J. M., G. Certain, C. Kraan, and C. F. Dormann. 2014. Stacking species archipelagos. Proceedings. Biological sciences / The Royal Society biotic interaction and environmental constraints at the biogeographical scale. Ecography 39:921-931. decomposition approaches to beta diversity via similarity and differentiation measures. Methods in Ecology and Evolution 7:919-928. 
433 Chase, J. M., N. J. B. Kraft, K. G. Smith, M. Vellend, and B. D. Inouye. 2011. Using

$434 \quad$ null models to disentangle variation in community dissimilarity from variation in $435 \quad \alpha$-diversity. Ecosphere 2:art24.

436 Chave, J., and E. G. Leigh. 2002. A spatially explicit neutral model of $\beta$-diversity in tropical forests. Theoretical Population Biology 62:153-168.

438 Chen, X.-Y., and F. He. 2009. Speciation and endemism under the model of island 439 biogeography. Ecology 90:39-45.

440 Condit, R., N. Pitman, E. G. Leigh Jr., J. Chave, J. Terborgh, R. B. Foster, P. Nunez 441 V., et al. 2002. Beta-diversity in tropical forest trees. Science 295:666-669.

442 Connolly, S. R., T. P. Hughes, and D. R. Bellwood. 2017. A unified model explains commonness and rarity on coral reefs. Ecology Letters 20:477-486.

444 Connor, E. F., and D. Simberloff. 1978. Species number and compositional similarity 445 of the Galapagos Flora and Avifauna. Ecological Monographs 48:219-248. 1979. The assembly of species communities: chance or competition?

$447 \quad$ Ecology 60:1132.

448 Diamond, J. M. 1969. Avifaunal equilibria and species turnover rates on the Channel Islands of California. Proceedings of the National Academy of Sciences 64:571975. Assembly of species communities. Pages 342-444 inEcology and Evolution of communities. Harvard University Press.

453 Diamond, J. M., and R. M. May. 1977. Species turnover rates on islands: dependence 
on census interval. Science 197:266-270.

455 Fukami, T., and M. Nakajima. 2011. Community assembly: alternative stable states or alternative transient states? Ecology Letters 14:973-984.

457 Gascuel, F., F. Laroche, A. S. Bonnet-Lebrun, and A. S. L. Rodrigues. 2016. The effects of archipelago spatial structure on island diversity and endemism: predictions from a spatially-structured neutral model. Evolution 70:2657-2666.

460 Gianuca, A. T., S. A. J. Declerck, P. Lemmens, and L. De Meester. 2017. Effects of dispersal and environmental heterogeneity on the replacement and nestedness components of $\beta$-diversity. Ecology 98:525-533.

463 Gotelli, N. J., and W. Ulrich. 2012. Statistical challenges in null model analysis. Oikos 121:171-180.

465 Graham, C. H., and P. V. A. Fine. 2008. Phylogenetic beta diversity: linking ecological and evolutionary processes across space in time. Ecology Letters $11: 1265-1277$.

468 Grainger, T. N., and B. Gilbert. 2016. Dispersal and diversity in experimental metacommunities: linking theory and practice. Oikos 125:1213-1223.

470 Gravel, D., F. Massol, E. Canard, D. Mouillot, and N. Mouquet. 2011. Trophic theory 471 of island biogeography. Ecology Letters 14:1010-1016.

472 Hanski, I. 2009. The theories of island biogeography and metapopulation dynamics.

473 Pages 186-213 in J. B. Losos and R. E. Ricklefs, eds. The theory of island 474 biogeography revisited. Princeton University Press, Princeton. 
475 Hanski, I., A. Moilanen, T. Pakkala, and M. Kuussaari. 1996. The quantitative incidence function model and persistence of an endangered butterfly metapopulation. Conservation Biology 10:578-590.

Helm, A., I. Hanski, and M. Pärtel. 2006. Slow response of plant species richness to habitat loss and fragmentation. Ecology Letters 9:72-77.

Holt, R. D. 2009. Toward a trophic island biogeography. Pages 143-185 in J. B. Princeton University Press, Princeton.

Hubbell, S. P. 2001. The unified neutral theory of biodiversity and biogeography. Princeton University Press, Princeton.

485 2009. Neutral theory and the theory of island biogeography. Pages 263-292 revisited. Princeton University Press, Princeton.

Hui, C., and M. A. McGeoch. 2014. Zeta diversity as a concept and metric that unifies Incidence-based biodiversity patterns. The American Naturalist 184:684-694. Biogeography Theory predict the scaling of functional trait composition with habitat area and isolation. Ecology Letters 20:135-146.

493 Jost, L. 2007. Partitioning diversity into independent alpha and beta components. Ecology 88:2427-2439.

495 Kadmon, R., and O. Allouche. 2007. Integrating the effects of area, isolation, and 
habitat heterogeneity on species diversity: a unification of island biogeography and niche theory. The American Naturalist 170:443-454.

Kalyuzhny, M., and N. M. Shnerb. 2017. Dissimilarity-overlap analysis of community dynamics: opportunities and pitfalls. Methods in Ecology and Evolution 0:1-10.

Kessler, D. A., and N. M. Shnerb. 2015. Generalized model of island biodiversity. Physical Review E - Statistical, Nonlinear, and Soft Matter Physics 91:1-11.

Ko, C. Y., O. J. Schmitz, and W. Jetz. 2016. The limits of direct community modeling approaches for broad-scale predictions of ecological assemblage structure. Biological Conservation 201:396-404.

Kraft, N. J. B., L. S. Comita, J. M. Chase, N. J. Sanders, N. G. Swenson, T. O. Crist, J. C. Stegen, et al. 2011. Disentangling the drivers of diversity along latitudinal and

508 Latombe, G., C. Hui, and M. A. McGeoch. 2017. Multi-site generalised dissimilarity modelling: using zeta diversity to differentiate drivers of turnover in rare and widespread species. Methods in Ecology and Evolution 8:431-442.

511 Legendre, P. 2014. Interpreting the replacement and richness difference components of beta diversity. Global Ecology and Biogeography 23:1324-1334.

513 Legendre, P., and M. De Cáceres. 2013. Beta diversity as the variance of community data: dissimilarity coefficients and partitioning. Ecology Letters 16:951-963.

515 Leibold, M. A., M. Holyoak, N. Mouquet, P. Amarasekare, J. M. Chase, M. F. 
multi-scale community ecology. Ecology Letters 7:601-613.

518 Leprieur, F., P. A. Tedesco, B. Hugueny, O. Beauchard, H. H. Dürr, S. Brosse, and T. Oberdorff. 2011. Partitioning global patterns of freshwater fish beta diversity reveals contrasting signatures of past climate changes. Ecology Letters 14:325334. decline revealed on the Hawaiian archipelago. Nature 543:710-713.

524 MacArthur, R. H., and E. O. Wilson. 1967. The theory of island biogeography. Princeton University Press, Princeton. resolves an underappreciated source of confusion in calculating species turnover. Ecology 98:933-939. metrics and null models: a review with new methods and software. Ecography 40:461-477.

Morrison, L. W. 2017. Insular plant turnover across a 22-year interval: a critical retrospective of the roles of pseudoturnover and cryptoturnover. Journal of Biogeography 44:1007-1017.

535 Nekola, J. C., and P. S. White. 1999. The distance decay of similarity in biogeography and ecology. Journal of Biogeography 26:867-878.

537 O’Dwyer, J. P., J. K. Lake, A. Ostling, V. M. Savage, and J. L. Green. 2009. An 
integrative framework for stochastic, size-structured community assembly. Proceedings of the National Academy of Sciences 106:6170-6175.

540 O’Dwyer, J. P., A. Rominger, and X. Xiao. 2017. Reinterpreting maximum entropy in 541 ecology: a null hypothesis constrained by ecological mechanism. Ecology $542 \quad$ Letters 20:832-841.

543 Ojima, M. N., and L. Jiang. 2017. Interactive effects of disturbance and dispersal on $544 \quad$ community assembly. Oikos 126:682-691.

545 Patiño, J., R. J. Whittaker, P. A. V. Borges, J. M. Fernández-Palacios, C. Ah-Peng, M. B. Araújo, S. P. Ávila, et al. 2017. A roadmap for island biology: 50 fundamental questions after 50 years of The Theory of Island Biogeography. Journal of Biogeography 44:963-983.

549 Pigot, A. L., and R. S. Etienne. 2015. A new dynamic null model for phylogenetic community structure. Ecology Letters 18:153-163.

551 Podani, J., and D. Schmera. 2011. A new conceptual and methodological framework for exploring and explaining pattern in presence - absence data. Oikos 120:16251638.

554 Qian, H., S. Chen, L. Mao, and Z. Ouyang. 2013. Drivers of $\beta$-diversity along 555 latitudinal gradients revisited. Global Ecology and Biogeography 22:659-670.

556 Qian, H., X. Wang, and Y. Zhang. 2012. Comment on "Disentangling the drivers of diversity along latitudinal and elevational gradients.” Science 335:1573-1573.

558 Rodrigues, J. F. M., and J. A. F. Diniz-Filho. 2017. Dispersal is more important than 
climate in structuring turtle communities across different biogeographical realms. Journal of Biogeography 44:2109-2120.

561 Rosindell, J., and L. J. Harmon. 2013. A unified model of species immigration, extinction and abundance on islands. Journal of Biogeography 40:1107-1118.

563 Rosindell, J., S. P. Hubbell, F. He, L. J. Harmon, and R. S. Etienne. 2012. The case for ecological neutral theory. Trends in Ecology and Evolution 27:203-208. sheds light on the zone of radiation. Ecology Letters 14:552-560.

569 Segre, H., R. Ron, N. De Malach, Z. Henkin, M. Mandel, and R. Kadmon. 2014. community assembly. Ecology Letters 17:1400-1408.

Si, X., A. Baselga, F. Leprieur, X. Song, and P. Ding. 2016. Selective extinction drives taxonomic and functional alpha and beta diversities in island bird assemblages. Journal of Animal Ecology 85:409-418.

575 Simberloff, D. S. 1969. Experimental zoogeography of islands: a model for insular colonization. Ecology 50:296-314.

577 Simberloff, D. S., and E. O. Wilson. 1969. Experimental zoogeography of islands: the colonization of empty Islands. Ecology 50:278-296.

579 Simberloff, D. S., and E. O. Wilson. 1970. Experimental zoogeography of islands: a 
two-year record of colonization. Ecology 51:934-937.

581 Stegen, J. C., A. L. Freestone, T. O. Crist, M. J. Anderson, J. M. Chase, L. S. Comita, H. V. Cornell, et al. 2013. Stochastic and deterministic drivers of spatial and temporal turnover in breeding bird communities. Global Ecology and Biogeography 22:202-212. Ecography 33:2-22. .2010b. A diversity of beta diversities: straightening up a concept gone awry. statistical inferences in null model analyses of diversity patterns. Global Ecology and Biogeography 26:1-5.

597 Valente, L. M., A. B. Phillimore, and R. S. Etienne. 2015. Equilibrium and non-equilibrium dynamics simultaneously operate in the Galápagos islands. Ecology Letters 18:844-852.

600 Veech, J. A., and T. O. Crist. 2007. Habitat and climate heterogeneity maintain 

Biogeography 16:650-656.

Wang, Y., Y. Bao, M. Yu, G. Xu, and P. Ding. 2010. Nestedness for different reasons: the distributions of birds, lizards and small mammals on islands of an inundated lake. Diversity and Distributions 16:862-873.

606

Wang, Y., V. Millien, P. Ding, and A. Algar. 2016. On empty islands and the small-island effect. Global Ecology and Biogeography 25:1333-1345.

608

Warren, B. H., D. Simberloff, R. E. Ricklefs, R. Aguilée, F. L. Condamine, D. Gravel, H. Morlon, et al. 2015. Islands as model systems in ecology and evolution: prospects fifty years after MacArthur-Wilson. Ecology Letters 18:200-217.

611 Weigelt, P., W. Jetz, and H. Kreft. 2013. Bioclimatic and physical characterization of 110:15307-15312.

614 Weigelt, P., M. J. Steinbauer, J. S. Cabral, and H. Kreft. 2016. Late Quaternary climate change shapes island biodiversity. Nature 532:99-102.

616 Whittaker, R. H. 1960. Vegetation of the Sisiyou Mountains, Orgeon and California. $617 \quad$ Ecological Monographs 30:279-338.

618 Whittaker, R. H. 1965. Dominance and diversity in land plant communities. Science 147:250-260.

620 Whittaker, R. J., K. A. Triantis, and R. J. Ladle. 2008. A general dynamic theory of 621 oceanic island biogeography. Journal of Biogeography 35:977-994. 
622 Wu, L., X. Si, R. K. Didham, D. Ge, and P. Ding. 2017. Dispersal modality

623 determines the relative partitioning of beta diversity in spider assemblages on

624 subtropical land-bridge islands. Journal of Biogeography 44:2121-2131.

625 Xiao, X., D. J. McGlinn, and E. P. White. 2015. A strong test of the Maximum

626 Entropy Theory of Ecology. The American Naturalist 185:E70-E80.

627 Xiao, X., J. P. O’Dwyer, and E. P. White. 2016. Comparing process-based and

628 constraint-based approaches for modeling macroecological patterns. Ecology

$62997: 1228-1238$.

630 Zillio, T., I. Volkov, J. R. Banavar, S. P. Hubbell, and A. Maritan. 2005. Spatial

631 scaling in model plant communities. Physical Review Letters 95:1-4.

632 
633 Table 1. Models fitted to the presence-absence patterns of 93 bird species in 42

634 islands in the Thousand Island Lake, China.

\begin{tabular}{lllllll}
\hline Model & \multicolumn{1}{c}{ A AIC } & AUC & $\begin{array}{l}\text { Number of } \\
\text { parameters }\end{array}$ & Richness & Dissimilarity & Turno \\
& & & & & & $R^{2}$ \\
non-neutral.GLM & -2627 & 0.95 & 97 & 0.91 & 0.37 & 0.53 \\
neutral.GLM & -172 & 0.61 & 5 & 0.90 & 0.01 & 0.45 \\
neutral.exponential & -8 & 0.57 & 2 & 0.41 & 0.01 & 0.17 \\
neutral.inverse ratio & 1587 & 0.60 & 1 & 0.69 & 0.04 & 0.46 \\
Total observation =3906 & & & & & & \\
\hline
\end{tabular}

635 Note: For the neutral exponential model: $c=\exp (-a I)$ and $e=\exp (-b A)$. For the

636 neutral inverse ratio model: $c=\frac{a}{I}$ and $e=\frac{b}{A}$. Dissimilarity is the sum of turnover

637 component and nestedness component. $\triangle \mathrm{AIC}$ are calculated by subtracting the AIC of

638 the null model (GLM with only one intercept) from the AIC of the fitted model. AUC

639 are calculated with a Mann-Whitney U statistic. 
641 Figure 1. Expected pairwise beta diversity of two identical islands (same colonization

642 and extinction rates), conditioning on both islands having species $(i>0, j>0)$.

643 Results are derived from the joint presence-absence distribution of two islands and

644 shown for different mainland pool sizes. Blue dashed line in panel (a) is the analytical

645 solution $E($ Jaccard.dissimilarity $)=\frac{2 \theta}{1+2 \theta}$ for the case of at least one island having

646 species $(N>0)$. Relative extinction rate is the ratio of extinction rate and colonization

647 rate.

648

649 Figure 2. Expected pairwise Jaccard beta diversities of two islands differing in

650 colonization and/or extinction rates, conditioning on both islands having species $(i>0$,

$651 j>0$ ). The blue dashed line in panel (a), (e) and (i) are the analytical solutions of

$652 E($ Jaccard.dissimilarity $)=\frac{(1+z) \theta}{1+(1+z) \theta}$, conditional on at least one island having

653 nonzero species. Expectations are provided for different levels of $z$, i.e. the number of

654 times relative extinction rate on the second island exceeds that on the first. The black

655 dashed lines in panel (d), (h) and (1) represent a 1:1 ratio. Different lines in a graph

656 represent different mainland pool sizes (for color legend see Fig. 1).

658 Figure 3. Empirical evaluation of the theoretically fitted alpha and beta diversity 659 patterns for the Thousand Islands Lake bird dataset. (a) - (1) are predictions of three 660 neutral models, (m) - (p) are predictions of a non-neutral model. Solid black lines 
bioRxiv preprint doi: https://doi.org/10.1101/292490; this version posted March 30, 2018. The copyright holder for this preprint (which was not certified by peer review) is the author/funder. All rights reserved. No reuse allowed without permission.

661 represent a 1:1 relationship and dashed red lines are fits of ordinary linear regressions,

662 Point densities from high to low are color-coded from red to blue. 
Figure 1
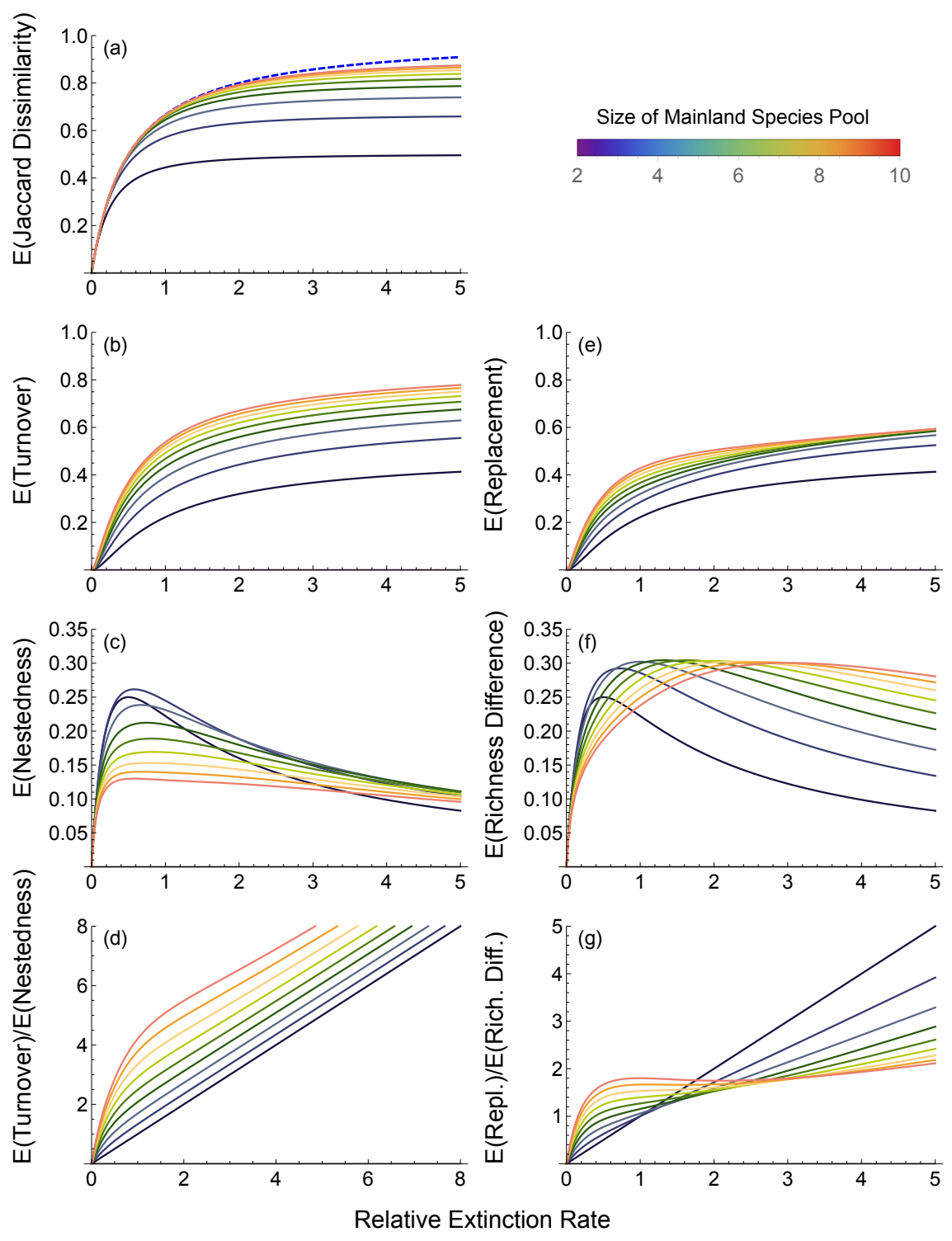
Figure 2
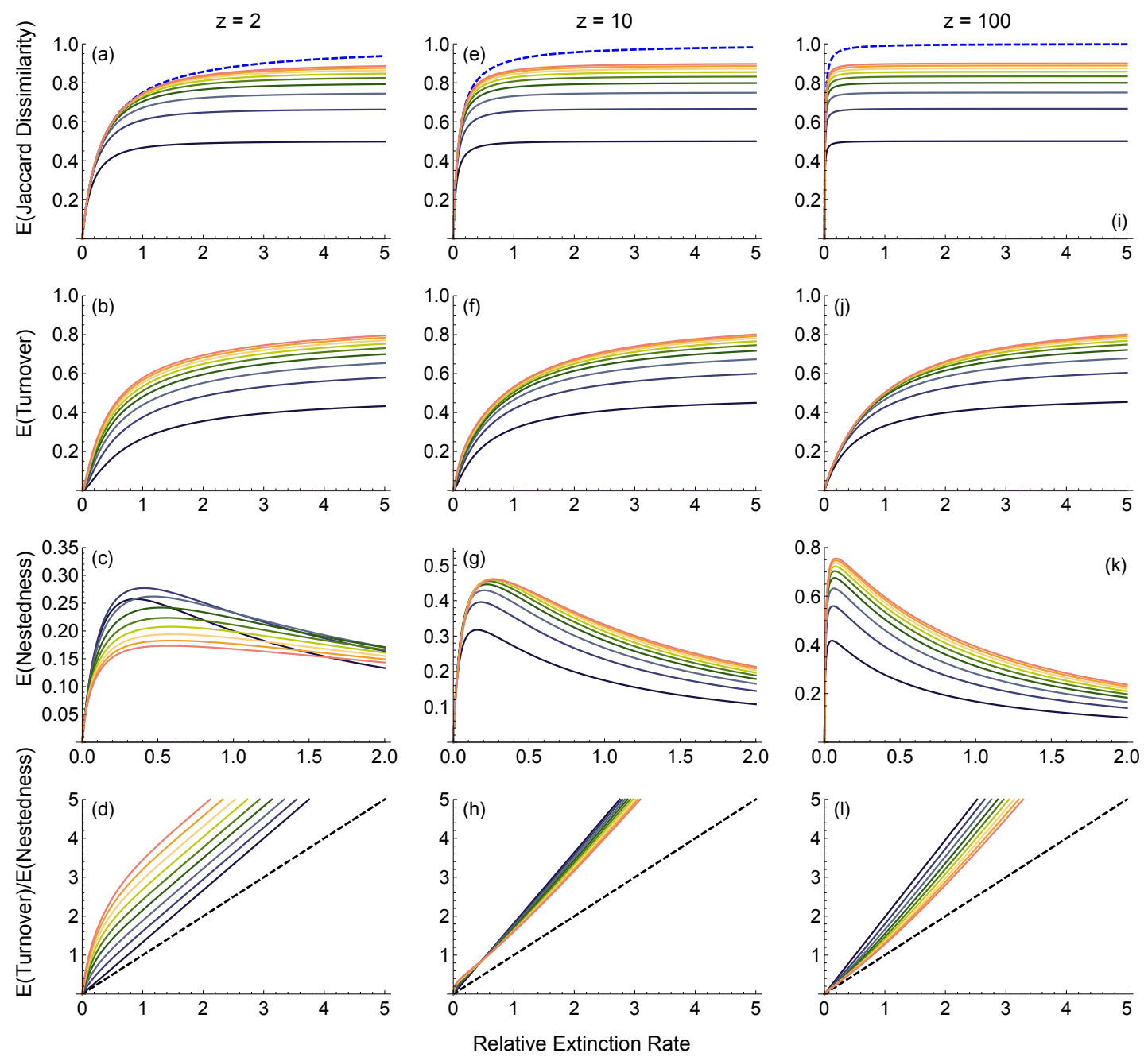
Figure 3
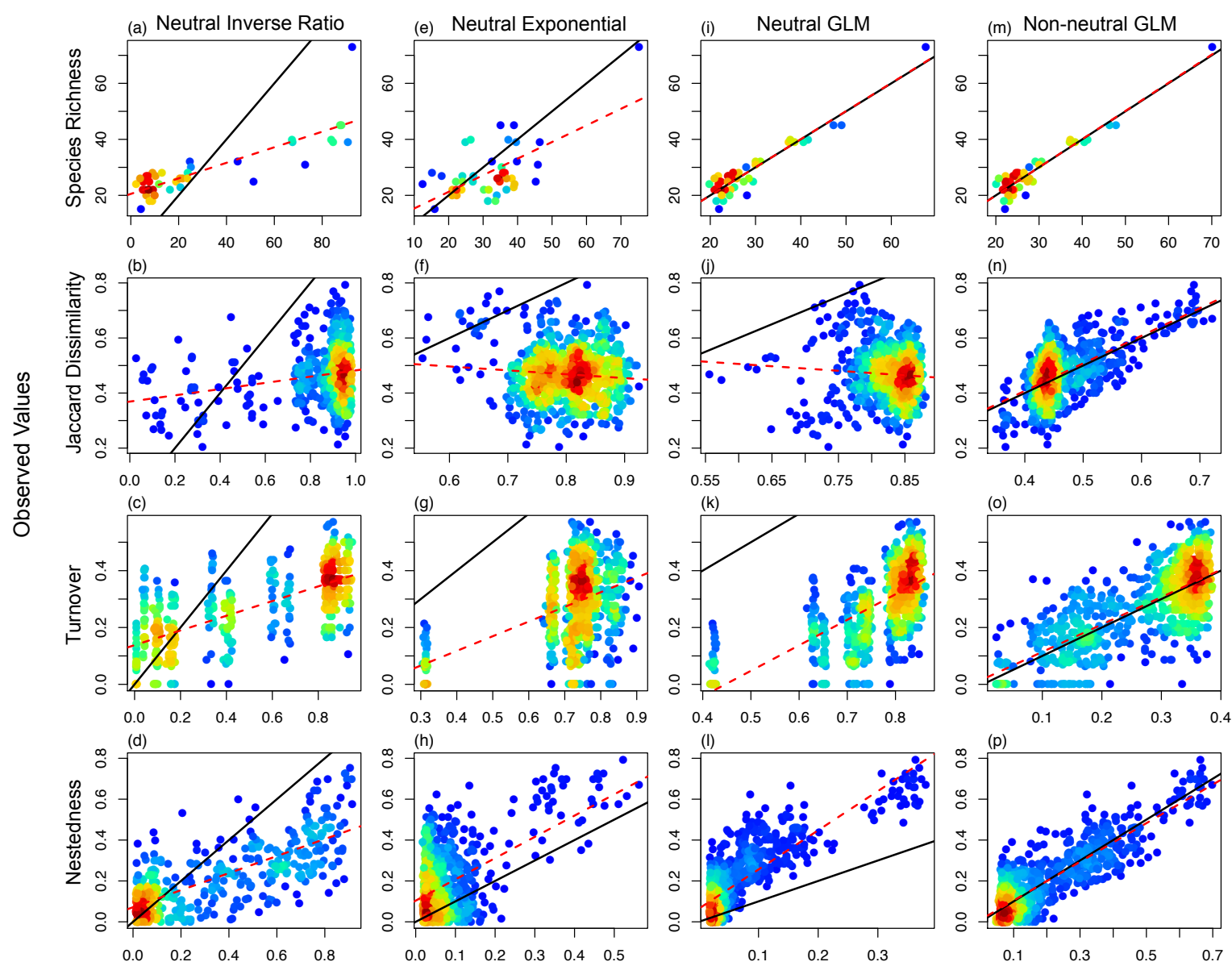

Predicted Values 